\title{
Supporting Information: A Mid-Infrared Spectroscopic Method for the Identification and Quantification of Dissolved Oil Components in Marine Environments
}

\author{
Robert Stach $^{1,2}$, Bobby Pejcic ${ }^{{ }^{*}}$, Emma Crooke$e^{1}$, Matthew Myers ${ }^{1}$ and Boris Mizaikoff ${ }^{2}$ \\ ${ }^{1}$ CSIRO, Energy, 26 Dick Perry Ave, Kensington, WA, 6151, Australia. \\ ${ }^{2}$ University of Ulm, Institute of Analytical and Bioanalytical Chemistry, Albert-Einstein-Allee 11, 89081 Ulm, Germa- \\ ny. \\ *Corresponding author: E-mail: Bobby.Pejcic@csiro.au Tel.: +61-8-6436-8814
}

\begin{abstract}
We give detailed information about the validation via GC-MS and the corresponding concentrations of BTEX contaminants within spiked seawater samples. All GC-MS measurements were performed by the National Measurement Institute (Perth, Western Australia).
\end{abstract}

Validation using gas chromatography. The BTEX compounds were determined on a HP $6890 \mathrm{GC} /$ Agilent 5973 MSD with an EST Encon purge-and-trap concentrator. Helium was bubbled through the water sample and the vapor was swept through a sorbent trap (Vocarb 3000). A ID HP-624 column was used with conditions as follows: split ratio of 50 to 1 ; initial column temperature was $40^{\circ} \mathrm{C}$; final column temperature was $245^{\circ} \mathrm{C}$; column flow at $1.0 \mathrm{ml} / \mathrm{min}$ in $\mathrm{He}$; and program column rate was $14^{\circ} \mathrm{C} / \mathrm{min}$. Naphthalene was liquid/liquid extracted with dichloromethane and analyzed on a HP 6890 GC / Agilent 5973 inert MSD. A J\&W DB-5MS (30 m x $0.25 \mathrm{~mm} \times 0.5 \mathrm{~mm})$ column was used and the conditions are as follows: splitless mode; injector temperature was $300^{\circ} \mathrm{C}$; and injector volume was $1 \mu$. Concentrations were determined by comparison with standards using electronic integration. Table S-1 shows the results for unspiked seawater samples. The results for diesel and crude oil spiked seawater are shown in tables S-2 and S-3. 
Table S-1. Summary of GC-MS results for unspiked seawater samples

Molfetta Qys

(Fuel Wharf) Conc. [ppb]

Analyte:

\begin{tabular}{ll}
\hline Benzene & $<1.0$ \\
Toluene & $<1.0$ \\
Ethylbenzene & $<1.0$ \\
o-Xylene & $<1.0$ \\
m,p-Xylene & $<1.0$ \\
Naphthalene & $<1.0$
\end{tabular}

Fremantle

South Beach Conc. [ppb]

Analyte:

Benzene $\quad<1.0$

Toluene

$<1.0$

Ethylbenzene $\quad<1.0$

o-Xylene $\quad<1.0$

m, p-Xylene $\quad<1.0$

Naphthalene $\quad<1.0$ 
Table S-2. Summary of GC-MS results in crude oil spiked seawater.

\begin{tabular}{lll}
$\begin{array}{ll}\text { 10o } \mu \mathrm{l} \\
\text { Analyte: }\end{array}$ & Conc. [ppb] & Error [ppb] \\
\hline Benzene & 10 & 1 \\
Toluene & 170 & 34 \\
Ethylbenzene & 70 & 13 \\
o-Xylene & 140 & 31 \\
m,p-Xylene & 380 & 84 \\
Naphthalene & 85 & 24
\end{tabular}

\begin{tabular}{lll}
$\begin{array}{l}500 \mu \mathrm{l} \\
\text { Analyte: }\end{array}$ & Conc. [ppb] & Error [ppb] \\
\hline Benzene & 31 & 4 \\
Toluene & 560 & 112 \\
Ethylbenzene & 140 & 27 \\
o-Xylene & 300 & 66 \\
m,p-Xylene & 750 & 165 \\
Naphthalene & 250 & 70
\end{tabular}

\begin{tabular}{lll}
$\begin{array}{lll}\text { 10oo } \mu \mathrm{l} \\
\text { Analyte: }\end{array}$ & Conc. [ppb] & Error [ppb] \\
\hline Benzene & 78 & 9 \\
Toluene & 1000 & 200 \\
Ethylbenzene & 200 & 38 \\
o-Xylene & 420 & 92 \\
m,p-Xylene & 1000 & 220 \\
Naphthalene & 330 & 92
\end{tabular}

\begin{tabular}{lll}
$\begin{array}{ll}\text { 6o ml } \\
\text { Analyte: }\end{array}$ & Conc. [ppb] & Error [ppb] \\
\hline Benzene & 380 & 46 \\
Toluene & 1900 & 380 \\
Ethylbenzene & 280 & 53 \\
o-Xylene & 590 & 130 \\
m,p-Xylene & 1400 & 308 \\
Naphthalene & 340 & 95
\end{tabular}

Table S-3. Summary of GC-MS results in diesel spiked seawater.

\begin{tabular}{|c|c|c|c|c|c|}
\hline $\begin{array}{l}100 \mu \mathrm{l} \\
\text { Analyte }\end{array}$ & Conc. [ppb] & Error [ppb] & $\begin{array}{l}500 \mu \mathrm{l} \\
\text { Analyte }\end{array}$ & Conc. [ppb] & Error [ppb] \\
\hline Benzene & 3,2 & 0,4 & Benzene & 4,8 & 1 \\
\hline Toluene & 52 & 10 & Toluene & 75 & 15 \\
\hline Ethylbenzene & 25 & 5 & Ethylbenzene & 28 & 5 \\
\hline o-Xylene & 53 & 31 & o-Xylene & 82 & 66 \\
\hline m,p-Xylene & 90 & 20 & m,p-Xylene & 110 & 24 \\
\hline Naphthalene & 53 & 15 & Naphthalene & 70 & 20 \\
\hline $\begin{array}{l}1000 \mu \mathrm{l} \\
\text { Analyte }\end{array}$ & Conc. [ppb] & Error [ppb] & $\begin{array}{l}\text { 6o ml } \\
\text { Analyte }\end{array}$ & Conc. [ppb] & Error [ppb] \\
\hline Benzene & 11 & 1 & Benzene & 83 & 10 \\
\hline Toluene & 140 & 28 & Toluene & 310 & 62 \\
\hline Ethylbenzene & 50 & 10 & Ethylbenzene & 75 & 14 \\
\hline o-Xylene & 110 & 92 & o-Xylene & 140 & 130 \\
\hline m,p-Xylene & 170 & 37 & m,p-Xylene & 230 & 51 \\
\hline Naphthalene & 110 & 31 & Naphthalene & 120 & 34 \\
\hline
\end{tabular}

